\title{
Teilzeitausbildung
}

\section{Pflegeberuf und Familie vereinbaren}

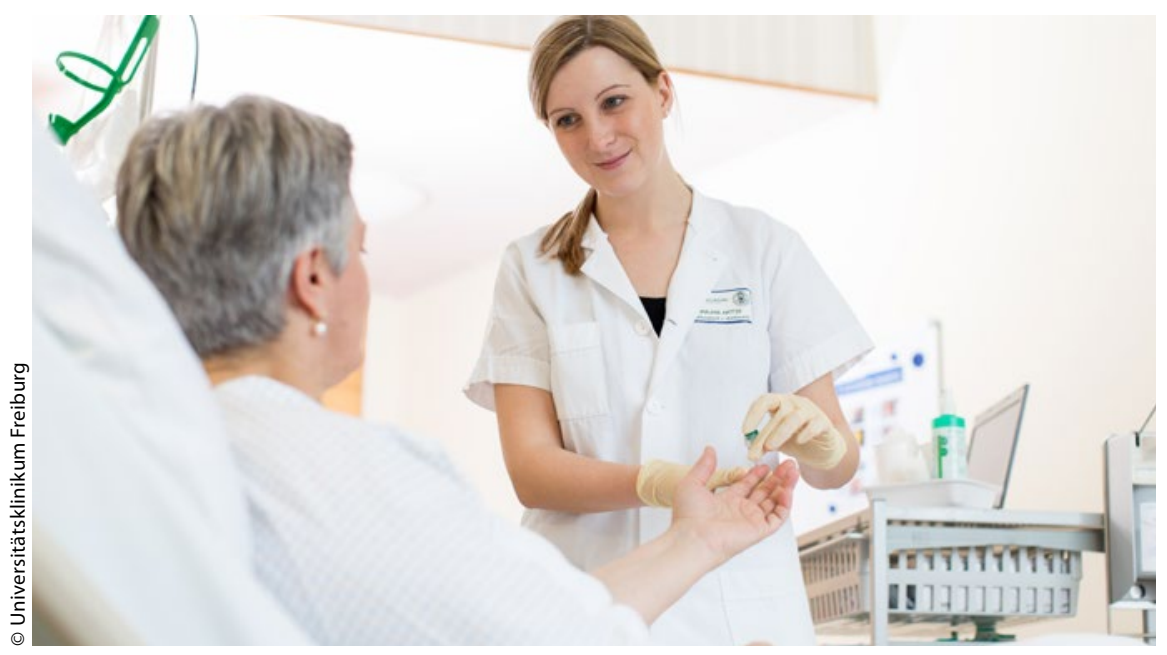

Praxisnahes Lernen: Bei der neuen Pflege-Ausbildung in Teilzeit lernen die angehenden Gesundheits- und Krankenpfleger früh den Umgang mit den Patienten.

— An der Akademie für Medizinische Berufe des Universitätsklinikums Freiburg wird zum Ausbildungsjahr 2016/2017 mit Beginn im November eine Teilzeitausbildung zu Gesundheits- und Krankenpflegerinnen und -Pflegern angeboten. Das Angebot einer solchen Teilzeitausbildung gibt es in Deutschland bisher nur vereinzelt. „Wir reagieren mit dem Angebot auf die sich verändernden Gesellschaftsstrukturen, in denen das Interesse an Teilzeitangeboten steigt", sagt Franz-Josef Overhoff, Leiter der Akademie für Medizinische Berufe am Uni- versitätsklinikum Freiburg. Die Ausbildung dauert vier Jahre und umfasst einen täglichen Aufwand in Theorie oder Praxis von etwa sechs Stunden. Vergütet wird die Teilzeitausbildung mit $75 \%$ des üblichen Ausbildungsgehaltes. Die Teilzeitausbildung zu Gesundheits- und Krankenpflegerinnen und -Pflegern richtet sich speziell an Eltern mit betreuungspflichtigen Kindern, Menschen mit pflegebedürftigen Angehörigen oder Wiedereinsteiger.

www.uniklinik-freiburg.de

\section{Fachweiterbildung}

\section{Intensivkrankenschwester werden}

— Das Karrierekonzept des medizinischen Personaldienstleisters competence \& more ermöglicht Gesundheits- und Krankenpflegern die Weiterbildung zur hochqualifizierten Pflegefachkraft für Intensivmedizin und Anästhesie. Bei der Umsetzung des Karrierekonzepts wird mit renommierten Partnern wie der Akademie der Gesundheit Berlin/ Brandenburg e.V., der Charité und dem HELIOS Klinikum Berlin-Buch zusammen gearbeitet. Eine Fachweiterbildung in der Intensivmedizin und Anästhesie dauert zwei Jahre und kann berufsbegleitend absolviert werden. Sie besteht aus Theorieund Praxismodulen, die sich über mehrere
Wochen am Stück erstrecken können. Während dieser Phasen steht der Mitarbeiter seinem Arbeitgeber nicht oder nur eingeschränkt zur Verfügung. Diesen Ausfall kann nicht jede Einrichtung kompensieren. Das Besondere am Karrierekonzept Fachweiterbildung ist die garantierte Lohnfortzahlung, indem Mitarbeiter flexibel eingesetzt werden. So gelingt es dem Unternehmen, den examinierten Pflegekräften die notwendige Zeit für die Teilnahme an den Modulen einzuräumen.

www.competenceandmore.de
Bachelor

\section{Intensivierte} Fachpflege

— Die in den letzten Jahren gestiegenen Ansprüche an die fachliche Qualifikation des Pflegepersonals machen innovative Maßnahmen zur Weiterentwicklung und Akademisierung der Pflegeberufe notwendig. Die Rheinische Fachhochschule Köln gGmbH bietet in Kooperation mit der Weiterbildungsstätte am Bildungszentrum des Universitätsklinikums Bonn den neuen Bachelorstudiengang „Intensivierte Fachpflege“ an. Der Studiengang richtet sich insbesondere an Gesundheits- und Krankenpfleger/innen, die Ihre Karriere mit einem Bachelorabschluss ausbauen möchten. Ziel des Studiengangs ist es, zum einen ergänzende berufliche Perspektiven für Pflegende zu schaffen und zum anderen durch qualifiziertes und motiviertes Personal zu einer Verbesserung der Patientenversorgung beizutragen. Das Studium vertieft medizinisches und pflegerisches Basiswissen und vermittelt betriebswirtschaftliche und medizinrechtliche Grundkenntnisse. Die Absolventen werden so adäquat auf eine Führungsposition im Krankenhaus oder in einer anderweitigen Einrichtung im Gesundheitswesen vorbereitet. Die Besonderheit des Studiengangs ist, dass Fachgesundheits- und Krankenpfleger/-innen Intensivpflege und Anästhesie, Operationsdienst, Pflege in der Onkologie, Pflege in der Psychiatrie oder Gesundheits- und Kinderkrankenpfleger/-in für Pädiatrische Intensivpflege und Anästhesie sowie Pflege in der Pädiatrischen Onkologie aufgrund der Studiengangsstruktur die ersten drei Semester anerkannt bekommen und somit den Bachelor of Science in nur vier Semestern erlangen können.

www.rfh-koeln.de 